\title{
Hybrid Model for Energy Consumption Forecasting in Buildings Stocks at Tropical Regions
}

\author{
Jose A. Bello A. ${ }^{1}$, Jimeno A. Fonseca ${ }^{2}$, Hugo Franco T. ${ }^{3}$ \\ ${ }^{1-3}$ Universidad Central, Bogota, Colombia \\ ${ }^{2}$ Future Cities Laboratory, Singapore-ETH Centre, ETH Zurich, Singapore \\ ${ }^{1}$ jbelloa1, ${ }^{3}$ hfrancot@ucentral.edu.co \\ 2ºnseca@arch.ethz.ch
}

\begin{abstract}
Electric energy consumption forecasting is a relevant issue to design and implement public policies related to energy generation and distribution at urban scale. This problem has been addressed from different standpoints, including traditional time series analysis and machine learning techniques, focused on the prediction of future energy demand according to metered data. This work proposes a hybrid model, based on computer simulation results from the City Energy Analyst toolbox and metered environmental and energy consumption data from a representative set of buildings located in Singapore. Such model is intended to provide reliable energy demand forecasts by using regression models. Three different regression methods were evaluated: a traditional SARIMA model and both NARX and Recurrent neural network architectures. The results obtained using this approach point out that RNN models provide accurate forecasts for 1 and 24 hours, outperforming NARX based models, while the SARIMA is, in general, unable to represent the electrical demand time series patterns.
\end{abstract}

\section{Introduction}

An appropriate forecasting of energy demand at the urban scale could provide valuable information to optimize the generation and distribution of electric energy in urban regions and to prevent inefficiencies and undesired conditions, such as electricity rationing or blackouts [Fisher-Vanden et al. (2012)]. Another application of proper forecasts regards to urban planning for city expansion or renovation. Indeed, the prediction of energy demand patterns of future buildings could allow preventive actions [Sallam and Malik (2018)]. Several works in the literature have addressed the problem of electric energy consumption forecasting in buildings at the urban scale from different perspectives, including building performance simulation [Pickering et al. (2018); Haydari et al. (2007)], machine learning techniques [Ahmad et al. (2017); Ruiz et al. (2016); Rahman et al. (2018); Ringwood et al. (2001)], and physics-based models [Fon- seca et al. (2016); Yamamoto et al. (2018)]. Some approaches apply modeling and simulation techniques to reproduce the physical interactions between building structure and technology, users and the environment [Santamouris et al. (2001)]. The forecasting methods in these works frequently rely on metered electric energy consumption time-series, while some of them also include external or explanatory features. A hybrid model that combines metered and simulated data to obtain a better performance on the forecast has not been tried, as far as the authors know. In this work, we use the simulation results of a multi-physics urban energy system model [Fonseca et al. (2016)] and smart meter measures of energy consumption into a machine learning model for time series analysis and forecasting. To evaluate the method performance, we tested a SARIMA model and two different machine learning approaches: autoregressive neural network models with exogenous variables (NARX), and recurrent neural networks (RNN). This hybrid model is intended to enhance the representation of complex energy consumption patterns to provide reliable forecasts, according to the specific building function (housing, industry, commerce, etc.), with potential applications to the design and optimization of electric energy generation and distribution systems.

\section{Seasonal Autoregressive Moving Average}

The Seasonal Autoregressive Integrated Moving Average (SARIMA) model was proposed by [Box and Jenkins (1976)] as a stochastic approach for regression and forecasting of time series exhibiting seasonal periodicity. The ARIMA family of models look for a linear combination of past values and errors of the time series. SARIMA models include a representation of the time series seasonal behavior by adding an extra linear combination of coefficients related to the seasonal period of the time series under consideration. The number of terms for each of the components -stationary and seasonal- is estimated by examining the autocorrelation $(\mathrm{ACF})$ and partial autocorrelation (PACF) functions. Thus, the model is defined by six parameters and a seasonal length value: $(\mathrm{p}, \mathrm{d}, \mathrm{q})(\mathrm{P}, \mathrm{D}, \mathrm{Q}) \mathrm{S}$, where the lowercase variables correspond to the stationary component and the uppercase 
variables to the seasonal component; 'p' refers to the number of auto-regressive (AR) terms, 'd' is the number of differences taken in the time series, ' $\mathrm{q}$ ' is the number moving average (MA) and ' $S$ ' represents the season period length. A general SARIMA model is written as:

$$
\begin{gathered}
\Phi_{P} B^{S} \phi_{p} B\left(1-B^{S}\right)^{D}(1-B)^{d} y_{t}=\Theta_{Q} B^{S} \theta_{q} B w_{t} \\
\Phi_{P}\left(B^{S}\right)=\left(1-\Phi_{1}\left(B^{S}\right)-\ldots-\Phi_{P}\left(B^{P S}\right)\right. \\
\phi_{p}(B)=\left(1-\phi_{1}(B)-\ldots-\phi_{p}\left(B^{p}\right)\right. \\
\Theta_{Q}\left(B^{S}\right)=\left(1-\Theta_{1}\left(B^{S}\right)-\ldots-\Theta_{P}\left(B^{P S}\right)\right. \\
\theta_{q}(B)=\left(1-\theta_{1}(B)-\ldots-\theta_{q}\left(B^{p}\right)\right.
\end{gathered}
$$

where (1) is the forecast equation, (2) is the autoregressive seasonal component, (3) is the autoregressive stationary component, (4) is the moving average seasonal component, and(5) is the moving average stationary component, with ' $B$ ', the backshift function, defined as $B^{k} y_{t}=y(t-k)$.

\section{Feed Forward Neural Networks}

Neural Network (NN) models have been used in a wide variety of fields [Ding et al. (2016); Angelova et al. (2015); Sutskever et al. (2014)]. Thanks to the rapid development of high computer power, and software packages for large-scale machine learning [Abadi et al. (2016)], NN can be used in a more practical way even with complex architectures such as convolutional $(\mathrm{CNN})$ or recurrent $(\mathrm{RNN})$. The $\mathrm{NN}$ consists in a set of "nodes" arranged in "layers". Depending on the network model, each node passes its output to other nodes in the network through weighted connections between them. The node output is calculated by an activation function [Rosenblatt (1961)] whose parameters are the node inputs (and a bias value in some cases):

$$
\hat{y}=\sigma\left(W^{T} X+b\right)
$$

where $X$ is the matrix of inputs, $W$ is the matrix of weights, $b$ is the vector of biases, $\sigma$ is the activation function (usually nonlinear) and $\hat{y}$ is the output. A set of nodes processing these calculations at the same time are called one "layer", and various layers can be stacked to increase the complexity of the network output. To train a NN, i.e. to minimize the error of the actual network output for a given input with respect to the expected output, it is necessary to correct the connection weights across the NN. A typical training (learning) algorithm iteratively uses the error of the network for an input in a training set to calculate a weight correction factor applied to each connection in the network, until the error obtained for every training input is below a target threshold. Such error is estimated by using loss functions, e.g. the Mean Squared Error (MSE) $e=\frac{1}{n} \sum_{i=1}^{n}(\hat{y}-y)^{2}$ The loss function is then minimized using an optimization algorithm, which updates the network weights:

$$
w_{i, j}=w_{i, j}-\alpha \frac{\delta e}{\delta w_{i, j}}
$$

where $w_{i, j}$ is the weight of the connection between nodes $\mathrm{i}$ and $\mathrm{j}, \alpha$ is a learning rate and the partial derivative represents the rate of change of the error with respect to the weight change for each connection. Every time that the whole training set has been presented to the network in the learning process, it is said that an "epoch" has been performed. It is common that a learning method requires several epochs to converge; however, modern learning approaches explore different epoch iteration numbers within parameter sweep processes to achieve an acceptable network behavior and define a preferable network parameterization, instead of using error thresholds. Indeed, several parameters can be tuned to reach a satisfying model, such as the number of nodes and/or layers, the transmission or activation function, the optimizer and its learning rate, the loss function, and the number of epochs, among others. There are several activation functions in the literature varying in their intervals, like the sigmoid function with range $[0,1]$, other operate within the range $[-1,1]$ like the tanh. Another one, like the Rectified Linear Unit, ReLU [Glorot et al. $(2011)]$, have a range between $[0, \infty)$.

$$
R e L U=\max (0, x)
$$

Recent works usually prefer the ReLU activation function, as well as the Adam optimizer [Kingma and $\mathrm{Ba}$ (2014)] which uses adaptative moment estimation for accelerating the learning process. Another tool to improve the learning convergence is the batch normalization [Ioffe and Szegedy (2015)], which incorporates a normalization step in each layer to reduce its internal covariate, looking for training and generalization enhancements.

\section{Nonlinear Autoregressive Neural Networks}

Different kind of inputs can be used in the model, such as explanatory features to predict the goal variable. In the case of time series forecasting, it is possible to include previous time steps of the time series (Ruiz et al., 3016). This method is known as a nonlinear autoregressive (NAR). If exogenous variables are considered in the model, it is called (NARX). The NAR family of models could overcome some limitations of the SARIMA approaches since they use nonlinear activation functions.

\section{Recurrent Neural Networks}

Recurrent Neural Networks (RNN) are a recent approach to time series prediction and sequence generation using NN [Karpathy et al. (2015); Lipton et al. (2015)]. This architecture consists in nodes with a set of gates that can recall their own previous time step outputs, as it is shown in Figure 1. Such architectures require an extra time dimension for each input. For time-series prediction, it is recommended that the time steps extend to one whole period at least. There are several RNN cell classes. The most popular is the Long Short-Term Memory, LSTM [Gers et al. (2000)], which can recall short- and long-term dependencies 
by the combination of gates [Olah (2015)]. There are two states as inputs for one node: hidden state and cell state. There is a set of four gate layers as shown in Figure 1.

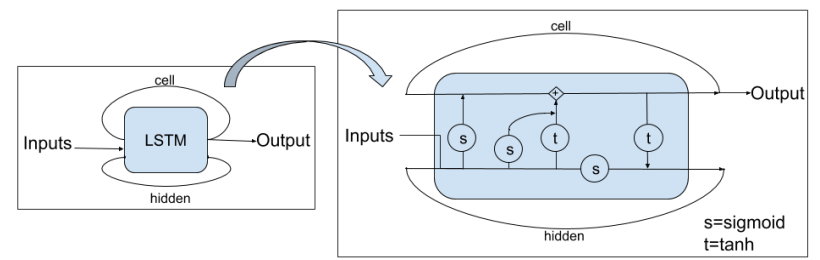

Figure 1: LSTM cell inner gates and operations. Source: Olah (2015), adapted with permission.

The "forget gate layer" takes as input the previous hidden state and the input features. This gate removes the unnecessary data by using a sigmoid activation function. Then, the "input gate layer" takes the previous hidden state and the input features as input and decides which values will be updated through a sigmoid activation function. In parallel, a new cell state candidate is computed with a tanh activation function. The "update gate layer" eliminates the data that the "forget gate layer" removed and updates the values of the cell state with the information passed by the "input gate layer". The "output gate layer" filters the updated cell state with a tanh activation function. It also filters the previous hidden state and the input features with a sigmoid activation function and combines the results into a single output.

\section{Methods}

This section describes the simulated and metered data used in this work and presents the hybrid model formulation for energy consumption forecasting, based on SARIMA, NARX and RNN regression methods.

\section{Data description}

The datasets in this work, used to design the hybrid model, belong to the CEA research team. The metered data were acquired in the facilities of the Nanyang Technological University (NTU) of Singapore, with a total of 40 buildings, while the simulated data were generated for the same buildings by the CEA team. There were four different sources of data:

- Weather data, obtained from a weather station at the NTU, with values measured in an hourly basis.

- Hourly electricity consumption of 40 buildings in the NTU.

- Geometric data (height, length, width, footprint, and proportions) from the sample buildings.

- Hourly simulated data of CEA for the 40 buildings in the NTU.

A fifth dataset represents the time features, such as hour of the day, day of the week and a weekend mark for Saturdays and Sundays. All data in the study correspond to acquisitions or simulations for one calendar year. The geometric building features were summarized in seven variables, created from their use percentage multiplied by their footprint. These features represent the footprint proportion for every use in each building. Table 1 presents these geometric variables.

Table 1: Geometric features used. Source: authors.

\begin{tabular}{|c|c|}
\hline Name & Description: Footprint \\
\hline fp.school & Proportion dedicated to school \\
\hline fp.office & Proportion dedicated to office \\
\hline fp.retail & Proportion dedicated to retail \\
\hline fp.restaurant & Dedicated to restaurant \\
\hline fp.multi_res & Dedicated to multiple residential \\
\hline fp.library & Proportion dedicated to library \\
\hline fp.lab & Proportion dedicated to lab \\
\hline
\end{tabular}

On the other hand, 8 features were removed from the weather dataset, since they had NULL values. Then, Pearson correlation tests were performed between the remaining variables to avoid highly similar inputs that could introduce some noise to the model. Most of the weather variables with high crossed correlation values (above 0.8 ) were represented closely related physical phenomena (e.g. radiation and illuminance), exhibiting small variations between them (e.g. horizontal, direct or diffuse), so that only the most representative variable for each group was included. Nevertheless, two extra variables, dry bulb temperature and relative humidity, were included despite they presented a high correlation, since they have different underlying physical phenomena. The weather features used to formulate the model are listed in Table 2.

The simulated data included features such as the number of people inside the building, indoor relative humidity, solar radiation incidence, sensible heat gained from transmission through the walls, among others. In this dataset, a total of 28 variables were available for the whole set of buildings. Pearson correlation tests were also performed between these variables; some of them had high correlation values and, as in the weather dataset, only the most representative variables were included. As a result, eight variables were selected (Table 3).

The target forecasting variable is the hourly electric energy consumption for each building (expressed in $\mathrm{kW} / \mathrm{h}$ ), whose ground truth corresponds to real

Table 2: Weather features used. Source: authors.

\begin{tabular}{|l|l|l|l|}
\hline Name & Description & Unit & \multicolumn{2}{|l|}{ Values } \\
\hline $\begin{array}{l}\text { Dry.Bulb. } \\
\text { Temp }\end{array}$ & $\begin{array}{l}\text { Temperature measured } \\
\text { in dry conditions }\end{array}$ & ${ }^{\circ} \mathrm{C}$ & $\begin{array}{l}-70 \quad \text { to } \\
70\end{array}$ \\
\hline $\begin{array}{l}\text { Relative Hu- } \\
\text { midity }\end{array}$ & Relative Humidity & $\%$ & $\begin{array}{l}0 \quad \text { to } \\
110\end{array}$ \\
\hline $\begin{array}{l}\text { Extraterres- } \\
\text { trial Hor- } \\
\text { izontal } \\
\text { Radiation }\end{array}$ & $\begin{array}{l}\text { Solar radiation re- } \\
\text { ceived on a surface } \\
\text { normal to the rays of } \\
\text { the sun at the top of } \\
\text { the atmosphere }\end{array}$ & & \\
\hline
\end{tabular}


Table 3: Simulated features used. Variables names are the same as in CEA. Source: CEA.

\begin{tabular}{|l|l|l|l|}
\hline Name & Description & Unit & Values \\
\hline People & People inside the building & & From 0 \\
\hline x_int & Indoor relative humidity & $\%$ & From 0 \\
\hline Eal_kWh & $\begin{array}{l}\text { Electricity consumption of } \\
\text { appliances and lights }\end{array}$ & $\frac{k W}{h}$ & From 0 \\
\hline $\begin{array}{l}\text { Qcs_sen_ } \\
\text { sys_kWh }\end{array}$ & $\begin{array}{l}\text { Total sensible cool de- } \\
\text { mand for all systems }\end{array}$ & $\frac{k W}{h}$ & From 0 \\
\hline $\begin{array}{l}\text { Qcs_lat_ } \\
\text { sys_kWh }\end{array}$ & $\begin{array}{l}\text { Total latent cool demand } \\
\text { for all systems }\end{array}$ & $\frac{k W}{h}$ & From 0 \\
\hline $\begin{array}{l}\text { Q_gain_sen_- } \\
\text { vent_kWh }\end{array}$ & $\begin{array}{l}\text { Sensible heat gain from } \\
\text { ventilation and infiltration }\end{array}$ & $\frac{k W}{h}$ & From 0 \\
\hline $\begin{array}{l}\text { I_sol_and } \\
\text { I_rad_kWh }\end{array}$ & Net radiative heat gain & $\frac{k W}{h}$ & From 0 \\
\hline T_int_C & Indoor temperature & ${ }^{\circ} \mathrm{C}$ & From 0 \\
\hline
\end{tabular}

metered data, as described above. The energy consumption patterns of four representative non-housing buildings for two weeks is shown in Figure 2.

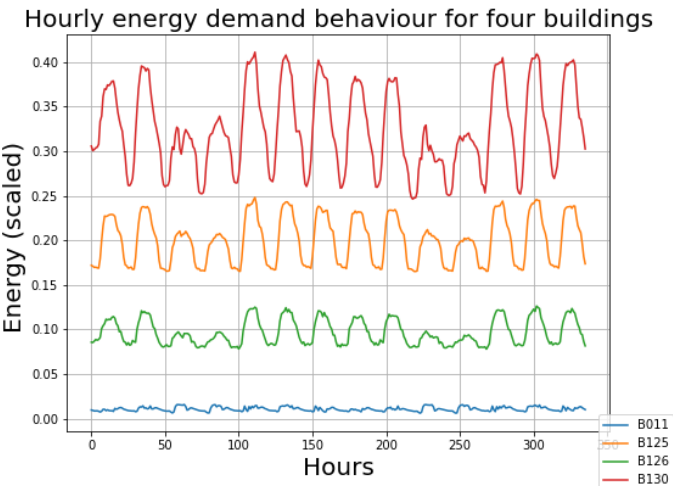

Figure 2: Scaled values for two-week energy consumption in a set of buildings. Source: own elaboration.

The demanded energy exhibits both daily and weekly seasonal behavior. The lower values in most of the time series corresponds to weekends and the five peak values to the maximum energy consumed during weekdays. Given Singapore is in a tropical region, there are no noticeable seasonality at other time scales.

\section{SARIMA implementation}

A traditional SARIMA approach was implemented. To determine the main periodicities, the energy consumption time series were plotted, and two seasonal periods were found: daily and weekly. The data was split in $70 \%$ entries for the training dataset and $30 \%$ for the testing dataset. Using the programming language ' $R$ ', the data were transformed into time series objects with a frequency of 24 hours by the ts function from base package. Both sets were scaled in training and forecasting steps with the Box-Cox transformation, implemented in the forecast package [Hyndman et al. (2005)]. This type of transformation needs a lambda parameter that was auto-determined by the BoxCox.lamba function.

In some cases, the time series need to be transformed to obtain a stationary behavior, and even with the seasonal time series, the seasonality needs to be transformed too. To get a transformed time series, a discrete differentiation step is used. The differentiation consists of subtracting two contiguous values of the time series and the result of each operation is the transformed time series. To define the number of required differences, the ndiffs and nsdiffs functions (from the forecast package) were used for nonseasonal and seasonal differences respectively. Once the differences were applied, the time series became stationary and non-seasonal. Then, the ACF and the PACF were plotted to determine, by visual inspection, the number of autoregressive and moving average terms for both non-seasonal, first few lags, and seasonal components, as the multiples of the season lags [Box and Jenkins (1976)]. Finally, with all the parameters needed to calculate the coefficients, the arima function from the mentioned package in ' $R$ ' was used to get the value of the model coefficients, using the same $70 \%$ of the data as training set. Once these values were obtained, the predicted values were calculated with the forecast function.

\section{NARX implementation}

To include the physical characteristics of the buildings that are constant along the year (such as the geometric features, represented by seven values per building), they were repeated for every hour of the year in each building dataset. All the inputs were scaled using the MinMaxScaler function from the scikit-learn package [Pedregosa et al. (2011)]. The data were split in $70 \%$ for the training dataset and $30 \%$ for the testing dataset. and shuffled with the train_test_split function, to guarantee that the training set has examples from different hours of the year. To include the AR terms in the model, defined three different numbers of previous values were used: zero (for 1-hour prediction), twelve and twenty-four. Once inputs, outputs and training and test sets were defined for each building, all these sets were stacked to obtain a general dataset for all the buildings in the study.

The number of inputs was used to define the number of nodes for each layer in the neural network: in one scenario the number of nodes per layer was equal to the number of inputs. Two more variations were made: one includes 10 extra nodes. Another one excludes 10 nodes from the initial number of inputs. The number of layers in the NARX model was defined according to the architecture proposed by [Ringwood et al. (2001)] and [Rahman et al. (2018)]. Then, five layers were chosen as the base model, and NN architectures defined by adding 3 layers to the base model, and by removing 3 layers from it, were also tested. All layers were normalized by using the batch_normalization function from the TensorFlow API [Abadi et al. (2016)] to increase the training speed, and the ReLU activation function was 
selected for all hidden layers. No activation function activation was used for the output layer. Following the work of [Goodfellow et al. (2016)], a random minibatch training was implemented to improve the training process convergence, with a batch size of 256 train samples. The number of epochs for all the scenarios was 300. The learning rate for the Adam optimizer was set to 0.003 and Mean Squared Error (MSE) was selected as loss function. Table 4 summarizes the NARX architecture parameters under evaluation.

Table 4: NARX model architecture parameters in this study. Source: own elaboration.

\begin{tabular}{|c|c|c|c|}
\hline AR input & Layers & Nodes & Output \\
\hline 0 & 2 & -10 & $\mathrm{t}$ \\
\hline$[\mathrm{t}-13, \mathrm{t}-1]$ & 5 & 0 & {$[\mathrm{t}, \mathrm{t}+24]$} \\
\hline$[\mathrm{t}-25, \mathrm{t}-1]$ & 8 & +10 & \\
\hline
\end{tabular}

\section{RNN implementation}

For the RNN model implementation, we used the TensorFlow API. The number of time steps ahead selected for the forecasting evaluation was se to 24 hours, so every feature was reshaped to include 24time steps in the time dimension. With this choice, there was the chance of making the output overlap on the 24-time step so, to make comparable the RNN forecasts with their NARX counterparts (where we generated the energy demand of every building in time-windows of 1 and 24-hours), the time steps of the output were overlapped 23 and 0 hours, generating 1 and 24 new hours ahead as outputs. The datasets were sorted properly for each building with the same proportions for training and testing sets (70\% for train, $30 \%$ for test), and stacked to include all buildings. The LSTM cell was selected for the RNN architectures. The number of cells or nodes was set based also on the number of input features, with the same variations as in the NARX exploration (by adding or removing 10 nodes), as well as the number of layers (2, 5 and 8 layers). The Adam optimizer and the MSE loss function were used to train the model with random mini-batches. Table 5 shows the architecture parameters used to build RNN models in this work.

Table 5: RNN model architecture parameters in this study. Source: own elaboration.

\begin{tabular}{|c|c|c|}
\hline Layers & Nodes & Output \\
\hline 2 & -10 & $\mathrm{t}$ \\
\hline 5 & 0 & {$[\mathrm{t}, \mathrm{t}+24]$} \\
\hline 8 & +10 & \\
\hline
\end{tabular}

\section{Models evaluation and comparison}

To evaluate the performance of all models in this study, the Mean Average Percentage Error (MAPE) was calculated for each forecasting window length.

$$
M A P E=\frac{100}{n} \sum_{i=1}^{n}\left|\frac{\hat{y}_{i}-y_{i}}{y_{i}}\right|
$$

In order to evaluate the performance of different neural network configurations (both NARX and RNN) using simulated data, two types of models were tested: one including the CEA simulated data as exogenous variables and other without including them. Thus, the combination of the parameters described in Tables 4 and 5 yield 108 models for NARX architectures and 36 models for RNN architectures. Every resulting model was used to forecast the building energy consumption 1 hour and 24 hours ahead. The time and weather features are the same for all buildings.

\section{Results}

\section{Dataset}

Depending on the NN model to evaluate, a given numbers of entries should be removed from the dataset: in the 1 hour ahead forecasting, the inputs belong to time $\mathrm{t}$ and the energy demanded to time $t+1$. In that way, one register is removed from the dataset. This procedure applies also for AR terms, where the number added to the forecasting window, in hours, implies removing that the same number of entries. This consideration also is employed for the RNN models. At the end, the number of training and testing samples (entries) varies in a range between 8712 and 8759 for each building (since the hours in a 365-day year are 8760).

\section{SARIMA}

The SARIMA model was run for each building individually because of the high variability among the building time series values between buildings prevented the training convergence of a generalized model. The number of stationary and seasonal terms for each building and the MAPE for daily and weekly forecasts are shown in Table 6.

Table 6: SARIMA parameters for one subset of buildings and performance in forecasting. Source: authors.

\begin{tabular}{|c|c|c|c|c|c|c|c|c|}
\hline & \multicolumn{3}{|c|}{ Stationary } & \multicolumn{3}{c|}{ Seasonal } & \multicolumn{2}{c|}{ MAPE } \\
\hline & $\mathrm{p}$ & $\mathrm{d}$ & $\mathrm{q}$ & $\mathrm{P}$ & $\mathrm{D}$ & $\mathrm{Q}$ & Day & Week \\
\hline $\mathrm{B} 082$ & 0 & 1 & 5 & 1 & 1 & 7 & 14.92 & 13.3 \\
\hline $\mathrm{B} 083$ & 0 & 1 & 5 & 1 & 1 & 7 & 14.97 & 13.34 \\
\hline $\mathrm{B} 087$ & 0 & 1 & 5 & 1 & 1 & 7 & 13.14 & 11.76 \\
\hline $\mathrm{B} 201$ & 4 & 1 & 0 & 5 & 1 & 3 & 0.04 & 0.05 \\
\hline B202 & 4 & 1 & 0 & 5 & 1 & 3 & 0.01 & 0.01 \\
\hline
\end{tabular}

Some groups of buildings need the same number of stationary and seasonal coefficients; however, the model performance exhibits a high variation between buildings with the same number of coefficients.

Table 7: SARIMA parameters for other set of buildings and performance in forecasting. Source: authors.

\begin{tabular}{|c|c|c|c|c|c|c|c|c|}
\hline & \multicolumn{3}{|c|}{ Stationary } & \multicolumn{3}{c|}{ Seasonal } & \multicolumn{2}{c|}{ MAPE } \\
\hline & $\mathrm{p}$ & $\mathrm{d}$ & $\mathrm{q}$ & $\mathrm{P}$ & $\mathrm{D}$ & $\mathrm{Q}$ & Day & Week \\
\hline B133 & 0 & 1 & 0 & 1 & 1 & 4 & 0.03 & 0.04 \\
\hline B084 & 2 & 1 & 3 & 2 & 1 & 4 & 315.09 & 111.03 \\
\hline B100 & 3 & 1 & 1 & 3 & 1 & 2 & 113.02 & 73.97 \\
\hline B125 & 7 & 1 & 3 & 2 & 1 & 2 & 0.02 & 0.04 \\
\hline
\end{tabular}

The performances shown in the Table 7 are quite inhomogeneous. The difference in the number of coeffi- 
cients is also considerable. Some buildings need more than ten autoregressive or moving average terms. Figures 3 and 4 show that the obtained SARIMA models provide acceptable forecasts for some buildings but has strong forecast deviations from real metered data for others. A small but noticeable forecasting lag is seen in the predicted value for a day in building B125 where the lowest energy real demanded occurs afterwards.
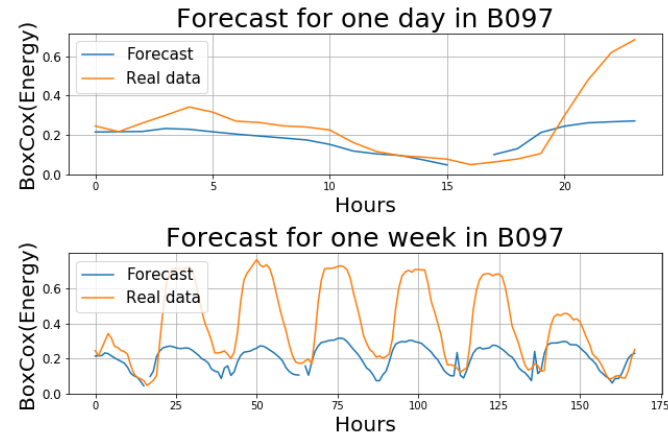

Figure 3: SARIMA forecast and real data comparison for building B09\%. Source: authors.
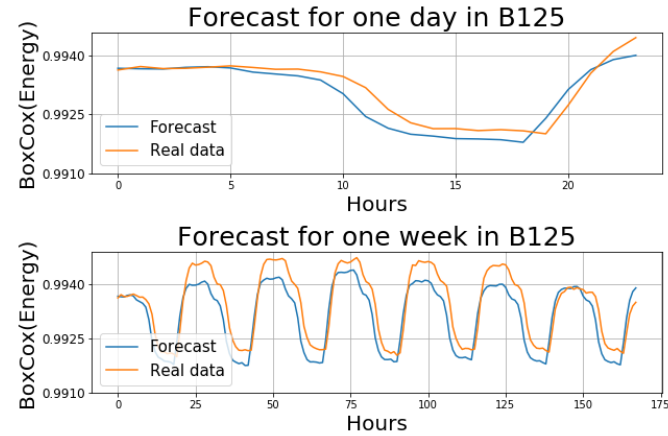

Figure 4: SARIMA forecast and real data comparison for building B125. Source: authors

\section{NARX}

As it was mentioned above, a total of 108 NARX models that were tested. The MAPE values for the models with the best performance using all buildings for train and test are listed in Table 8, for each forecasting window length (time-window, T.W.).

Table 8: NARX model performance for different architectures ( $A R=24$ for all models). Source: authors.

\begin{tabular}{|l|l|l|l|l|l|l|}
\hline \multirow{2}{*}{ T.W. } & \multirow{2}{*}{ Layer } & \multirow{2}{*}{ Node } & \multicolumn{2}{|c|}{ No CEA vars. } & \multicolumn{2}{c|}{ CEA vars. } \\
\cline { 4 - 7 } & & & Train & Test & Train & Test \\
\hline \multirow{2}{*}{$24 \mathrm{~h}$} & 2 & -10 & 697.8 & 700.4 & $\mathbf{5 0 8 . 4}$ & $\mathbf{5 0 6 . 3}$ \\
\cline { 2 - 7 } & 5 & 10 & $\mathbf{5 4 3 . 1}$ & $\mathbf{5 4 2 . 3}$ & 931.5 & 919.5 \\
\hline \multirow{2}{*}{$\mathrm{lh} \mathrm{h}$} & 2 & 0 & $\mathbf{9 . 7}$ & $\mathbf{9 . 7}$ & 13.1 & 12.8 \\
\cline { 2 - 7 } & 8 & 0 & 12.6 & 11.8 & $\mathbf{9 . 3}$ & $\mathbf{9 . 2}$ \\
\hline
\end{tabular}

The highlighted values represent the best NARX models for each time window. The 24-terms autoregressive architecture is the best forecasting configuration in most of the cases. For all the time windows, the models with CEA simulated variables get a better performance. This improvement in the model training is also observable by comparing error evolution and training and test comparison in Figures 5 and 6 ,
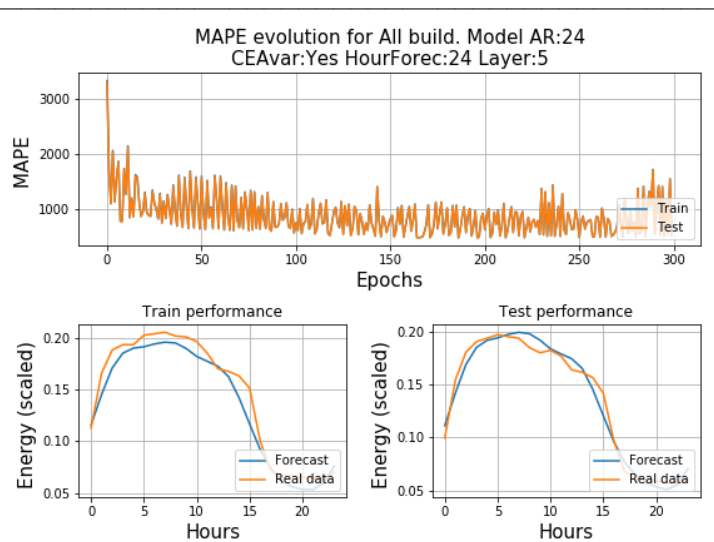

Figure 5: 24-hour forecast with CEA vars. Source: authors.
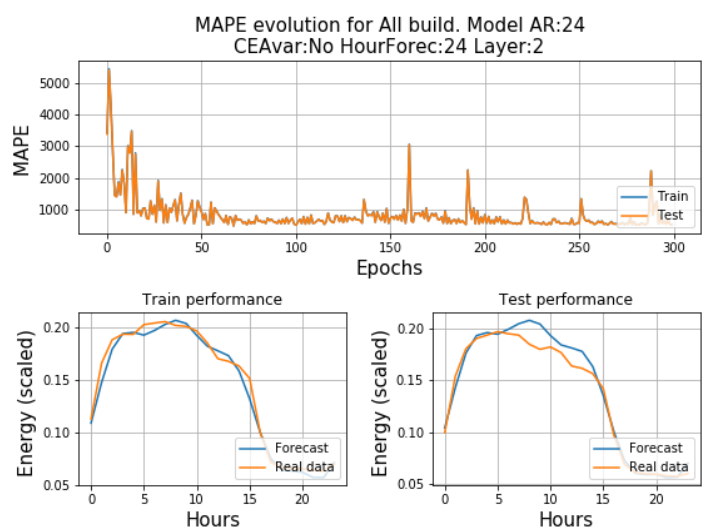

Figure 6: 24-hour forecast without CEA vars. Source: authors.

where the use of CEA variables enhances the learning process through successive epochs.

These models reached a good approximation to the energy consumption in both training and testing sets without evidence of overfitting. There is a noisy behavior in the evolution of training error, possibly caused by the random mini-batch training.

\section{RNN}

Table 9 presents the models with better performance, from the complete set of RNN 36 architectures, as a summary of the RNN model exploration.

Table 9: RNN model MAPE values for different architectures and hour forecast in all buildings. Source: authors.

\begin{tabular}{|l|l|l|l|l|l|l|}
\hline \multirow{2}{*}{ T.W. } & \multirow{2}{*}{ Layer } & \multirow{2}{*}{ Nodede } & \multicolumn{2}{|c|}{ no CEA vars. } & \multicolumn{2}{c|}{ CEA vars. } \\
\cline { 4 - 7 } & & & Train & Test & Train & Test \\
\hline \multirow{2}{*}{$24 \mathrm{~h}$} & 8 & 10 & 351.3 & 351.4 & $\mathbf{2 2 4 . 1}$ & $\mathbf{2 2 2 . 8}$ \\
\cline { 2 - 7 } & 8 & 10 & $\mathbf{3 2 1 . 3}$ & $\mathbf{3 2 0 . 3}$ & 229 & 227.8 \\
\hline \multirow{2}{*}{$1 \mathrm{~h} \mathrm{~S}$} & 5 & 10 & $\mathbf{3 3 7 . 2}$ & $\mathbf{3 3 7 . 3}$ & 213.5 & 214.2 \\
\cline { 2 - 7 } & 8 & 10 & 814.8 & 817 & $\mathbf{2 0 3}$ & $\mathbf{2 0 3 . 9}$ \\
\hline
\end{tabular}

The highlighted values represent the best RNN models for each forecasting time window. As in the NARX scenario exploration, most of the models with 10 extra nodes report better performances. For all the time windows, the models that include simulated variables get a better performance than their purely metered data counterparts. Figure 7 shows the training evolu- 
tion and representative 24-hour forecasts for the best RNN model.
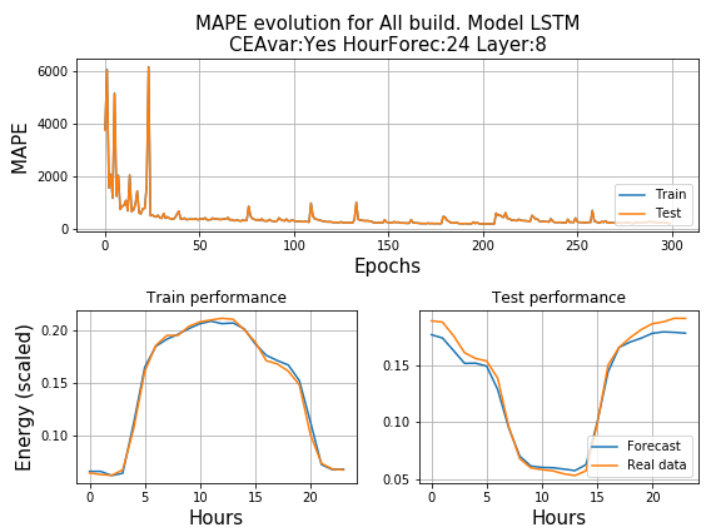

Figure \%: Error evolution and train and test comparison for RNN model forecast 24 hours in all buildings. Source: authors.

The error decay is smoother than it is for the NARX models, and a good fitting performance is rapidly achieved before 50 epochs. The forecast comparison proves that the training and testing processes exhibit a proper performance, accurately predicting the variations in the energy consumption real data.

\section{Discussion and conclusions}

Energy demand time series presented several challenging features related to the specific use of each building as well as the climatic conditions of their location (Singapore). However, the hybrid model proposed in this work (real and simulated data as input of a regression-based forecasting model) was able to provide a reliable energy consumption forecasting method by using both simulated and metered data beside a proper regression approach. The SARIMA model exhibited a poor performance and did not reach an acceptable fit of the time series for most of the buildings in the dataset, and sometimes presented large forecast deviations from the actual metered energy consumption data. Moreover, the resulting SARIMA model structure exhibited a high variation between buildings and required ten or more AR and/or MA terms (almost a half of the season length) for some cases. Indeed, there are considerable variations in the number of stationary and seasonal component coefficients, causing that every new building will require its own training process, including ACF and PACF analysis. Given such drawback, this model appears as an expensive alternative for a large quantity of buildings, since it lacks a unique, generalized structure to produce reliable forecasts for every new instance (building). The possible addition of exogenous variables to propose a SARIMAX approach could be an interesting research path to confirm such limitations. On the other hand, the NARX models explored in this study provide sound forecasts for all buildings. The inclusion of $\mathrm{AR}$ terms in the input features enhanced both the model training convergence and the forecasting performance. Architectures without AR terms cannot reach the performance levels achieved by their counterparts with 24 AR terms. The non-linear combination of explanatory variables and AR terms in the NN architecture could generalize the energy forecasting for the buildings in the dataset, with a reliable fitting of the real data. Including simulated variables from the CEA toolbox improved the NARX performance for most of the scenarios, with a MAPE reduction of about $6 \%$ in the 24 -hour forecasting and $5 \%$ in the 1-hour forecasting. Also, the number of epochs to achieve a good performance were much lower when the CEA variables were included. The RNN LSTM-based models performed even better than the NARX models for all forecasting scenarios, no matter the time-window selected for each test. A reduction of $55 \%$ in MAPE value of the best 24-hour forecasting model was achieved using LSTM instead NARX as regression approach. Most of the models in this study yields better predictions by adding extra nodes to each layer (up to 10 in the proposed experimentation) and if extra layers are included (up to 3 for this study), as it is shown in Tables 8 and 9. A combination of LSTM cells with CEA variables yields the best performance. The best model for 24-hour energy demand forecasting was an RNN with LSTM cells, 8 layers and 10 extra nodes including the CEA simulation variables. This architecture reduced the MAPE value in about $30 \%$ compared to the model without CEA variables. Additionally, models designed to forecast energy consumption for shorter periods have lower MAPE values. Thus, a more detailed performance exploration for different RNN configurations could lead to better predictions and should be addressed in future works.

\section{References}

Abadi, M., P. Barham, J. Chen, Z. Chen, A. Davis, J. Dean, M. Devin, S. Ghemawat, G. Irving, M. Isard, M. Kudlur, J. Levenberg, R. Monga, S. Moore, D. G. Murray, B. Steiner, P. Tucker, V. Vasudevan, P. Warden, M. Wicke, Y. Yu, and X. Zheng (2016). TensorFlow: A system for large-scale machine learning. pp. 21.

Ahmad, M. W., J.-L. Hippolyte, M. Mourshed, and Y. Rezgui (2017). Random Forests and Artificial Neural Network for Predicting Daylight Illuminance and Energy Consumption. pp. 7.

Angelova, A., A. Krizhevsky, and V. Vanhoucke (2015, May). Pedestrian detection with a LargeField-Of-View deep network. In 2015 IEEE International Conference on Robotics and Automation (ICRA), pp. 704-711.

Box, G. E. P. and G. Jenkins (1976). Time Series Analysis, Forecasting and Control. Holden-Day, Incorporated. 
Ding, N., C. Benoit, G. Foggia, Y. Bésanger, and F. Wurtz (2016, January). Neural Network-Based Model Design for Short-Term Load Forecast in Distribution Systems. IEEE Transactions on Power Systems 31(1), 72-81.

Fisher-Vanden, K., E. T. Mansur, and Q. J. Wang (2012, January). Costly blackouts? measuring productivity and environmental effects of electricity shortages. (17741).

Fonseca, J. A., T.-A. Nguyen, A. Schlueter, and F. Marechal (2016, February). City Energy Analyst (CEA): Integrated framework for analysis and optimization of building energy systems in neighborhoods and city districts. Energy and Buildings 113, 202-226.

Gers, F. A., J. Schmidhuber, and F. Cummins (2000, October). Learning to Forget: Continual Prediction with LSTM. Neural Computation 12(10), 2451-2471.

Glorot, X., A. Bordes, and Y. Bengio (2011, June). Deep Sparse Rectfier Neural Networks. Proceedings of the fourteenth international conference on artificial intelligence and statistics, 315-323.

Goodfellow, I., Y. Bengio, and A. Courville (2016). Deep Learning. MIT Press. http://www. deeplearningbook .org.

Haydari, Z., F. Kavehnia, M. Askari, and M. Ganbariyan (2007, October). Time-series load modelling and load forecasting using neuro-fuzzy techniques. In 2007 9th International Conference on Electrical Power Quality and Utilisation, pp. 1-6.

Hyndman, R. J., A. B. Koehler, J. K. Ord, and R. D. Snyder (2005, January). Prediction intervals for exponential smoothing using two new classes of state space models. Journal of Forecasting 24(1), 17-37.

Ioffe, S. and C. Szegedy (2015, February). Batch Normalization: Accelerating Deep Network Training by Reducing Internal Covariate Shift. arXiv:1502.03167 [cs]. arXiv: 1502.03167.

Karpathy, A., J. Johnson, and L. Fei-Fei (2015, June). Visualizing and Understanding Recurrent Networks. arXiv:1506.02078 [cs]. arXiv: 1506.02078 .

Kingma, D. P. and J. Ba (2014, December). Adam: A Method for Stochastic Optimization. arXiv:1412.6980 [cs]. arXiv: 1412.6980.

Lipton, Z. C., J. Berkowitz, and C. Elkan (2015, May). A Critical Review of Recurrent Neural Networks for Sequence Learning. arXiv:1506.00019 [cs]. arXiv: 1506.00019.
Olah, C. (2015). Understanding LSTM Networks colah's blog. http://colah.github.io/posts/2015-08Understanding-LSTMs/. Accessed: 2019-01-30.

Pedregosa, F., G. Varoquaux, A. Gramfort, V. Michel, B. Thirion, O. Grisel, M. Blondel, P. Prettenhofer, R. Weiss, V. Dubourg, J. Vanderplas, A. Passos, D. Cournapeau, M. Brucher, M. Perrot, and E. Duchesnay (2011). Scikit-learn: Machine Learning in Python. Journal of Machine Learning Research 12, 2825-2830.

Pickering, E. M., M. A. Hossain, R. H. French, and A. R. Abramson (2018, October). Building electricity consumption: Data analytics of building operations with classical time series decomposition and case based subsetting. Energy and Buildings 177, 184-196.

Rahman, A., V. Srikumar, and A. D. Smith (2018, February). Predicting electricity consumption for commercial and residential buildings using deep recurrent neural networks. Applied Energy 212, 372385 .

Ringwood, J. V., D. Bofelli, and F. T. Murray (2001, May). Forecasting Electricity Demand on Short, Medium and Long Time Scales Using Neural Networks. Journal of Intelligent and Robotic Systems 31(1), 129-147.

Rosenblatt, F. (1961, March). Principles of neurodynamics. perceptrons and the theory of brain mechanisms. (VG-1196-G-8).

Ruiz, L., M. Cuéllar, M. Calvo-Flores, M. Jiménez, L. G. B. Ruiz, M. P. Cuéllar, M. D. Calvo-Flores, and M. D. C. P. Jiménez (2016, August). An Application of Non-Linear Autoregressive Neural Networks to Predict Energy Consumption in Public Buildings. Energies 9(9), 684.

Sallam, A. A. and O. P. Malik (2018, November). Electric Distribution Systems. John Wiley \& Sons.

Santamouris, M., N. Papanikolaou, I. Livada, I. Koronakis, C. Georgakis, A. Argiriou, and D. N. Assimakopoulos (2001, January). On the impact of urban climate on the energy consumption of buildings. Solar Energy $70(3)$, 201-216.

Sutskever, I., O. Vinyals, and Q. V. Le (2014). Sequence to Sequence Learning with Neural Networks. In Z. Ghahramani, M. Welling, C. Cortes, N. D. Lawrence, and K. Q. Weinberger (Eds), Advances in Neural Information Processing Systems 27, pp. 3104-3112. Curran Associates, Inc.

Yamamoto, T., A. Ozaki, M. Lee, and H. Kusumoto (2018, January). Fundamental study of coupling methods between energy simulation and CFD. Energy and Buildings 159, 587-599. 